\title{
Gonococcal tysonitis
}

\author{
N. J. FIUMARA \\ From the Division of Communicable Venereal Diseases, Boston
}

Infection of Tyson's glands is not a common complication of gonorrhoea. Its incidence is not known, and neither the Center for Disease Control of the Public Health Service nor national statistical compilations of diseases can give details. Reports from urological services of university hospitals confirm that it is rare by such remarks as, 'I saw a case six years ago' etc. However, Bavidge (1976) reported two cases of gonococcal tysonitis with urethritis and one case of gonococcal tysonitis without urethritis in 1975 at the Newcastle General Hospital. I have had two cases in Massachusetts during the fiscal year 1976 from a total of 60000 visits in our venereal diseases clinics.

Tyson's glands are a pair of sebaceous glands located near the coronal sulcus, one on either side of the frenum; they produce smegma. When infected with the gonococcus, the glands manifest unilateral or bilateral tender swelling and on moderate pressure, pus may be expressed from ducts opening on either sides of the frenum. Usually the infection responds to antibiotics and the swelling subsides. If not, incision and drainage of the glands will be necessary followed by another course of antibiotics. Patients who also have a tight prepuce should be circumcised to prevent reinfection of these glands, should the patient contract another infection with the gonococcus.

\section{Case report}

A white single, heterosexual college student came to the clinic complaining of a painful swelling on either side of the frenum of about one month's duration. Four weeks earlier the patient had been treated for gonococcal urethritis with $3.5 \mathrm{~g}$ of ampicillin orally plus a simultaneous gulp of $1 \mathrm{~g}$ of probenecid at the college infirmary. There was then some swelling and tenderness of Tyson's glands but the pain and swelling subsided after treatment only to recur five to seven days later. As he was studying for final examinations, he delayed seeking further medical care until this admission.

Address for reprints: Dr N. J. Fiumara, Director, Division of Communicable Diseases, Massachusetts Department of Public Health, 600 Washington Street, Boston 0211, Massachusetts, USA Received for publication 8 November 1976
On examination the patient had no urethral discharge but he did have bilateral swelling and tenderness on both sides of the frenum. There was a halo of erythema at the ductal entrance and pus could readily be expressed even with minimum pressure (Figure). A smear of this pus revealed abundant Gram-negative intracellular diplococci and the culture on Thayer-Martin medium gave oxidase positive colonies 48 hours later.

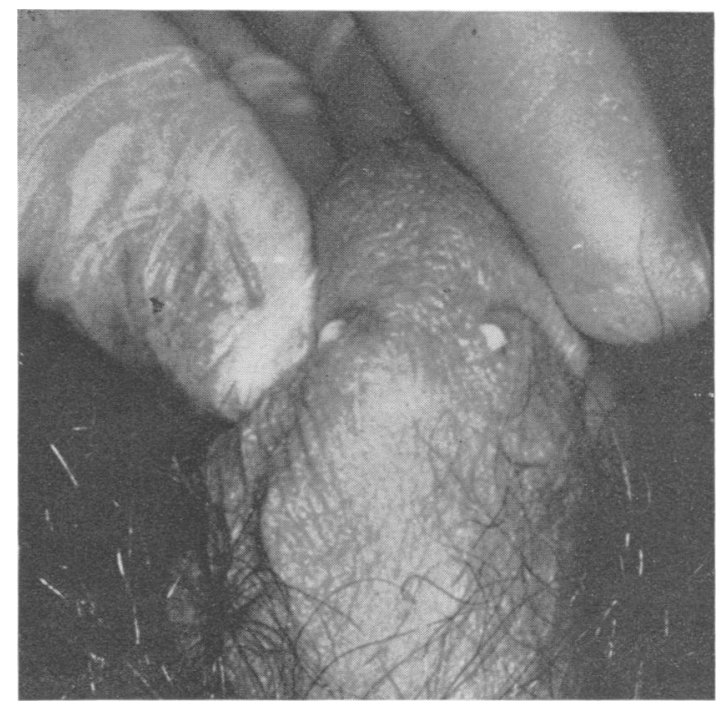

Figure Bilateral swelling on both sides of frenum

A calcium alginate swab specimen of the urethra was negative by smear and culture. The patient had no clinical evidence for early symptomatic syphilis and the blood Rapid Plasma Reagin Circle Card (RPR-CT) test was non-reactive. The patient was not allergic to penicillin and aqueous procaine penicillin G 4.8 million units was given intramuscularly and 2.4 million units administered daily for the next four days. The abscess was incised and drained with chunks of solidified smegma excised. The patient made an uneventful recovery and was discharged at the end of the week.

Reference

Bavidge, K. J. N. (1976). Gonococcal infection of the penis. British Journal of Venereal Diseases, 52, 66. 